\title{
Thermomechanical model for NiTi-based shape memory alloys covering macroscopic localization of martensitic transformation
}

\author{
M. Frosta,b,*, B. Benešováa ${ }^{a, c}$, H. Seiner ${ }^{a}$, M. Kružík ${ }^{d}$, P. Šittnere,b, P. Sedlák ${ }^{a, b}$ \\ ${ }^{a}$ Czech Academy of Sciences, Institute of Thermomechanics, Dolejškova 5, 18200 Prague, Czech Republic \\ ${ }^{b}$ Czech Academy of Sciences, Nuclear Physics Institute, Husinec - ̌̌ez 130, 250 68, R̆ež, Czech Republic \\ ${ }^{c}$ Department of Mathematical Analysis, Charles University, Sokolovská 83, 18600 Prague, Czech Republic \\ ${ }^{d}$ Czech Academy of Sciences, Institute of Information Theory and Automation, Pod Vodárenskou věži 4, 18200, \\ Prague, Czech Republic \\ ${ }^{e}$ Czech Academy of Sciences, Institute of Physics, Na Slovance 2, 18221 Prague, Czech Republic
}

\begin{abstract}
The work presents a thermomechanical model for polycrystalline NiTi-based shape memory alloys developed within the framework of generalized standard solids, which is able to cover loading-mode dependent localization of the martensitic transformation. The key point is the introduction of a novel austenite-martensite interaction term responsible for strain-softening of the material. Mathematical properties of the model are analyzed and a suitable regularization and a time-discrete approximation for numerical implementation to the finite-element method are proposed. Model performance is illustrated on two numerical simulations: tension of a superelastic NiTi ribbon and bending of a superelastic NiTi tube.
\end{abstract}

Keywords: NiTi shape memory alloys, constitutive modeling, localizaton, Mori-Tanaka method

\section{Introduction}

Having found many applications in medicine, civil engineering or aerospace industry (Mohd Jani et al., 2014), NiTi-based alloys are a prominent class within shape memory alloys (SMAs) usually utilized in the form of thin structures, e.g. wires, strips, tubes or plates. It is often experimentally observed that stress-induced martensitic transformation (mainly when it is induced by uniaxial tension) does not occur in a spatially homogeneous manner on a macroscopic scale; instead, localized "martensitic bands" appear within the austenitic sample and the transformation propagates by their multiplication and/or by movement of their fronts. The macroscopic picture is very similar to the localization of plastic deformation in certain steels and alloys well-known as Lüders bands: the onset of transformation is usually accompanied by a stress overpeak followed by a stress plateau, and the material rehardens after exhausting the available portion of transformation strain.

The simplest example of localization can be found in NiTi wires loaded in tension (Shaw and Kyriakides, 1995; Sedmák et al., 2016). A wider range of localization patterns can be observed in tensile deformation on NiTi thin strips as thoroughly reported by Shaw and Kvriakides (1997b,a). Their works also documented the strong dependence of localization patterns on the deformation rate. The dynamics of formation, propagation and coalescence of transformation bands on NiTi strips and the relation

${ }^{*}$ Corresponding author. Institute of Thermomechanics, Dolejškova 5, CZ-18200 Prague. Tel.: +420 266053712. Fax: +420286584695 .

Email address: mfrost@it.cas.cz (M. Frost) 
to the strain-rate was further studied (e.g. by Pieczyska et al., 2013; Zhang et al., 2010; Bian et al., 2018, and many others).

A further sample shape that can be easily manufactured and exhibits various localization patterns is the tube. Sun and Li (2002) were first to show spiral bands forming in tension on the surface of thin-walled superelastic NiTi tubes. The development of localized deformation in tension and homogeneity of deformation in compression were later documented by (Mao et al., 2010; Reedlunn et al., 2014; Bechle and Kyriakides, 2014). Bent tubes exhibit wedge-like localization patterns on the part of the surface deformed predominantly in tension whereas homogeneous deformation was detected on the part deformed predominantly in compression (Reedlunn et al., 2014; Bechle and Kyriakides, 2014). Experiments were also performed in multiaxial loading: propagating transformation fronts were observed in pure tension, they were absent in pure torsion, and progressive behavior in between these pure modes were observed in (Sun and Li, 2002; Reedlunn et al., 2017); in proportional biaxial loading experiments by Bechle and Kyriakides (2016), localized helical bands with inclinations dependent on the stress ratio formed except for the loading modes close to equibiaxial tension. More detailed review on experimental results on the localization and their relation to constitutive modeling challenges can be found in (Frost et al., 2018a).

It is expected that the key for understanding of formation of macroscopic martensitic bands are interactions at the mesoscopic level, i.e. the level of transforming grains (where the martensitic transformation is always localized) and their aggregates (Šittner et al., 2005). This motivated several in-situ microstructure studies focused on characterization of stress redistributions associated with martensite nucleation and growth (Young et al., 2010; Sedmák et al., 2016). A detailed picture of the martensitic band front in a loaded NiTi wire obtained by 3D X-ray synchrotron diffraction (Sedmák et al., 2016) shows that the internal stress states in grains massively change and redistribute within the propagating front so that, at the onset of transformation, austenitic grains were (in average) exposed to equivalent stresses more than $200 \mathrm{MPa}$ higher then externally measured plateau stress. The observed stress heterogeneity was rationalized with help of a FEM simulation assuming considerable strain-softening during progress of martensitic transformation. Since strainsoftening is "invisible" in macroscopic stress-strain curves with a plateau, dedicated experiments revealing the course of the strain-softening are needed. Quantitative results have been reached by Hallai and Kyriakides (2013) via a sophisticated measurement of a NiTi-stainless steel composite and, recently, by Alarcon et al. (2017) via a special geometry of NiTi bulk specimen.

Despite abundance of constitutive models of SMAs in the literature - originating at different scales of description, see recent reviews by Cisse et al. (2016a, b) - comprehensive three-dimensional macroscopic (continuum) models incorporating localization have been attempted rarely. Most frequently, simple isotropic plasticity-based models with the strain-softening are used to study localization in NiTi wires (Iadicola and Shaw, 2004; Badnava et al., 2014), strips (Shaw and Kvriakides, 1997a; Azadi et al., 2007) or tubes deformed in tension (Jiang et al., 2017b; Razaee-Hajidehi et al., 2019).

Involving the strain-softening into constitutive models brings inevitably problems with their regularity such as mesh-dependence of solution or spurious concentration of strain to infinitely small regions. These problems were already analyzed (motivated by their practical impact mainly in simulation of damage) and several regularization techniques were proposed (see e.g. Bažant and Jirásek, 2002; Jirásek and Rolshoven, 2003). They are based on so-called nonlocal continuum theories, in which response of a material point is not uniquely determined by values of state and internal variables (fields) in that point only, but the state of material points in its vicinity is also taken into account. A useful tool for incorporating such information is introduction of so-called non-local variable(s). Then, two issues have to be addressed: (a) defining how the nonlocal ("twin") variable is related to a local one(s), and (b) modifying the constitutive laws, which involve both local and non- 
local variables. Concerning the first point, two well-established approaches can be borrowed from structural mechanics: the implicit nonlocal gradient approach (iNGA) and the nonlocal integral approach (NIA). In iNGA, the local and corresponding nonlocal variables are linked via an additional (elliptic) partial differential equation; in NIA, these variables are linked via an integral relation - the nonlocal variable in a material point is defined as a weighted integral average of the values of the local one gained in a close neighborhood of that point. Corresponding general mathematical formulations are closely related, since iNGA can be derived from NIA using particular weighting functions (Peerlings et al., 2001). Both approaches naturally incorporate an internal parameter related to some characteristic length scale related to the extend of relevant neighborhood. The examples of iNGA in SMA models can be found in (Duval et al., 2011; Armattoe et al., 2014; Badnava et al., 2014), whereas NIA approach was employed e.g. in (Ahmadian et al., 2015; Sedmák et al., 2016).

Recently, a few more elaborated models which tried to capture also the loading mode-dependence of localization patterns observed in experiments appeared (Pouva et al., 2017; [Jiang et al., 2017a, b) . Namely, they attempt to capture the fact that whereas the transformation in tension is usually localized, the transformation in compression is observed to be homogeneous (Elibol and Wagner, 2015; Watkins et al., 2018) via heuristic modifications of the "yield criterion" used in the plasticitybased models.

In this work, we present an extension of our original SMA model formulated in the framework of generalized standard solids by Sedlák et al. (2012). We introduce a novel austenite-martensite interaction term and show the capability of the extended model to reproduce evolution of localized martensitic transformation in NiTi SMAs. The original model (Sedlák et al., 2012) is briefly summarized in Section 2. The derivation of the interaction term, which is based on the elastic energy of a material with misfitting inclusions by Mori and Tanaka (1973), is presented in Section 3 , The derivation of the interaction term is not limited to any particular loading mode emphasizing the ambition of the model to be used in general loading scenarios. Splitting the internal variables into local and non-local ones, which is done heuristically in the definition of the interaction energy, allows to rigorously perform the regularization of the complete model as presented in Section 4. Numerical implementation into finite element method (FEM) is described and illustrating simulations are performed in Section 5 .

\section{Local model}

Within this work, we develop a phenomenological constitutive model of the NiTi SMAs capable to capture the localization effects. The term phenomenological here means that the model aims to describe the polycrystalline material in some average sense and underlying microscopic features are taken into account through inner variables of the model. Such models allows for an easy implementation, less time consuming simulations and have the potential to be applied in industrial applications. Thus, a large number of such models has been proposed so far (e.g. Lagoudas et al., 2012; Stupkiewicz and Petrvk, 2013; Stebner and Brinson, 2013; Auricchio et al., 2014; Mehrabi et al., 2014; Gu et al., 2015; Chatziathanasiou et al., 2016, and many others).

Here, we adapt a three-dimensional macroscopic model for NiTi SMA proposed in (Sedlák et al., 2012) and further developed and validated in (Frost et al., 2014, 2016a, b; Sedmák et al., 2016; Frost et al., $2018 \mathrm{~b}$ ) that has been shown to perform well even for non-proportional loading. We review the model here for the reader's convenience, as we will extend it later in Section 3 to capture localization.

The modelled specimen is assumed to occupy, at the reference configuration, the domain $\Omega \subset \mathbb{R}^{3}$. As the primary state variable we choose the displacement of the specimen $u: \Omega \rightarrow \mathbb{R}^{3}$. The total 
strain tensor, $\boldsymbol{\varepsilon}(x)$, is related to the displacement via

$$
\varepsilon(x)=\frac{1}{2}\left(\nabla u(x)+(\nabla u(x))^{\top}\right),
$$

which is given locally in every material point $x \in \Omega$. As frequent in macroscopic modelling of SMAs (cf. Gu et al., 2015; Sadjadpour and Bhattacharya, 2007; Chemisky et al., 2011), we additionally introduce two internal variables: the scalar $\xi: \Omega \rightarrow \mathbb{R}^{3}$ representing the volume fraction of martensite and satisfying $\xi(x) \in[0,1]$ in every material point and a tensor variable, $\boldsymbol{\varepsilon}^{\text {tr }}: \Omega \rightarrow \mathbb{R}^{3}$, representing the transformation strain. With these two variables, we may write the the conventional small strain decomposition, assumed to be valid for every $x \in \Omega$, as

$$
\varepsilon^{\mathrm{el}}(x)=\varepsilon(x)-\xi(x) \varepsilon^{\mathrm{tr}}(x) .
$$

Here $\varepsilon^{\mathrm{el}}$ is the elastic strain and $\varepsilon^{\text {tr }}$ is a macroscopic variable used for storing information about microscopic internal structure of martensite. Later, particularly in the mathematical part, we will also use the inelastic strain defined via

$$
\varepsilon^{\mathrm{in}}(x)=\xi(x) \varepsilon^{\operatorname{tr}}(x) .
$$

Crystallographic considerations show (Otsuka and Wayman, 1998) that there exists a maximum value of strain that is attainable due to phase transformation; so, the transformation strain is considered to lie in a bounded convex set. In addition, the austenite-martensite transition is nearly volume preserving, so that it is justified to consider $\varepsilon^{\text {tr }}$ (as well as $\varepsilon^{\text {in }}$ ) to be trace-free tensors. For the model in hand, this means that for every material point $x \in \Omega$, we require

$$
\varepsilon^{\operatorname{tr}}(x) \in\left\{A \in \mathbb{R}^{3 \times 3}: A \text { is symmetric, } \operatorname{tr}(A)=0,\langle A\rangle \leq 1\right\},
$$

where $\operatorname{tr}(A)$ denotes the trace of a tensor $A$ and $\langle\cdot\rangle: \mathbb{R}^{3 \times 3} \rightarrow \mathbb{R}^{+}$is a suitable positively 1-homogeneous convex function; by a particular form of this function, tension-compression asymmetry is captured in the model.

It is convenient to formulate the model within the framework of so-called generalized standard solids (see Halphen and Nguyen, 1975). That means that we need to prescribe two scalar functions, the free energy $f_{T}\left(\varepsilon, \varepsilon^{\mathrm{tr}}, \xi\right)$, that depends on the state variables, as well as dissipation function $d_{T}\left(\varepsilon^{\operatorname{tr}}, \xi, \dot{\varepsilon}^{\operatorname{tr}}, \dot{\xi}\right)$, that depends both on the internal state variables and their rates. The free energy and the dissipation function may depend on the temperature $T$ that we, however, consider prescribed in the whole specimen (quasistatic approximation), which is indicated by the respective index.

Within this section, the free energy and dissipation function are understood to be given locally in each material point (so that all the variables actually depend on $x \in \Omega$ ), but for a better readability we suppress to indicate this from now on.

The free energy is given as a sum of elastic and chemical contributions:

$$
f^{T}\left(\varepsilon, \xi, \varepsilon^{\mathrm{tr}}\right)=\underbrace{\frac{1}{2}\left(\varepsilon-\xi \varepsilon^{\mathrm{tr}}\right): \mathbb{C}(\xi):\left(\varepsilon-\xi \varepsilon^{\mathrm{tr}}\right)}_{\text {elastic energy }}+\underbrace{\Delta s^{A M}\left(T-T_{0}\right) \xi}_{\text {chemical energy }},
$$

where $\mathbb{C}$ is the tensor of elastic constants, $s^{A M}$ is the specific entropy difference between the austenite and martensite phase and $T_{0}$ is the temperature at which austenite and martensite are (energetically) at equilibrium. 
Furthermore, we choose the dissipation function as

$$
d^{T}\left(\varepsilon^{\operatorname{tr}}, \xi, \dot{\varepsilon}^{\operatorname{tr}}, \dot{\xi}\right)= \begin{cases}\Delta s^{A M}\left[T_{0}-M_{\mathrm{s}}+\xi\left(M_{\mathrm{s}}-M_{\mathrm{f}}\right)\right]|\dot{\xi}|+\sigma^{\mathrm{reo}}(T)\left\|\xi \dot{\varepsilon}^{\operatorname{tr}}+\dot{\xi} \varepsilon^{\text {tr }}\right\| & \text { if } \dot{\xi} \geq 0, \\ \Delta s^{A M}\left[A_{\mathrm{f}}-T_{0}+\xi\left(A_{\mathrm{s}}-A_{\mathrm{f}}\right)\right]|\dot{\xi}|+\sigma^{\mathrm{reo}}(T)\left[\left\|\dot{\xi} \varepsilon^{\mathrm{tr}}\right\|+\left\|\xi \dot{\varepsilon}^{\operatorname{tr}}\right\|\right] & \text { if } \dot{\xi}<0,\end{cases}
$$

where $M_{\mathrm{s}}, M_{\mathrm{f}}$ as well as $A_{\mathrm{s}}, A_{\mathrm{f}}$ are the temperature at which the austenite-to-martensite (forward) as well as the martensite-to-austenite (reverse) start or finish, respectively. Moreover, $\sigma^{\text {reo }}$ is a constant (may depend on temperature) that characterizes the amount of dissipated energy due to reorientation.

We refer the reader to (Sedlák et al., 2012; Frost et al., 2016a) for a detailed derivation of the form of this dissipation function. Let us just mention at this point that the form is chosen in such a way that reflects the following ideas: during the forward transformation, the appearing martensite can reorient immediately and thus the appearance of martensite and its reorientation are fully coupled processes. On the other hand, the reverse transformation can only happen if the martensite can reorient to a form that can be coupled to austenite. Thus, a suitable reorientation of martensite must become favourable in order to allow the reverse phase transformation to occur.

\section{Non-local model}

In this section, we introduce an energy term which allows to capture localization into the constitutive model summarized in Section 2. We use micromechanics-inspired approach motivated by available (experimental) knowledge. The basic observations on localization of martensitic transformation in NiTi alloys can be summarized in the following points:

- Localization of martensitic transformation appears only in some loading modes. The localization was documented on NiTi wires, strips or tubes loaded in tension, but most of the experiments on loading in compression and shear reveal macroscopically homogeneous transformation (Elibol and Wagner, 2015; Watkins et al., 2018) 1

- The localization of martensitic transformation in tension cannot be explained as a sole result of geometry (cross-section) change associated with large deformation induced by transformation. Although the geometric changes, the decrease of the integral force due to necking in the transformed zone as well as the lack of material hardening 2 contribute to the observed asymmetry between the localized transformation in tension and the homogeneous one in compression, several recent experiments (Hallai and Kyriakides, 2013; Sedmák et al., 2016; Alarcon et al., 2017) clearly documented a considerable stress decrease in the material during progression of martensitic transformation in tension. The decrease of stress depends on the particular material microstructure, and in magnitude, it could be even comparable with the transformation plateau stress.

- The transformation stress in tension does not decrease linearly with the extent of transformation (martensite volume fraction). Experiments suggest the decrease is maximal at the beginning of the transformation, and then it continuously attenuates during further transformation (Hallai and Kyriakides, 2013; Alarcon et al., 2017).

\footnotetext{
${ }^{1}$ The situation in torsion of NiTi tubes is ambiguous. Observations by Sun and Li (2002) suggest homogeneity of transformation, whereas detailed SEM analysis in (Peng et al., 2008) reveals mesoscopic heterogeneity in martensite distribution in the form of microscopic martensitic lamellae. Nevertheless, the heterogeneities are on a much finer scale compared to those occurring during tensile loading.

${ }^{2}$ We recall here the well-known Considère criterion.
} 
- On the level of single grains forming the polycrystalline material, the martensitic transformation spreads heterogeneously. Bands of martensite running through austenitic grains are observed during stress-induced transformation (Otsuka and Wayman, 1998). The reason for the strainsoftening can be thus sought in the interaction of fully martensitic regions with the surrounding elastic austenite.

- On the other hand, temperature induced transformation in polycrystalline samples does not occur at a single transformation temperature but in a broader temperature interval. This suggests there is a distribution of transformation temperatures within the polycrystalline material, and, consequently, also a distribution of transformation stresses. Moreover, differently oriented grains can fulfill transformation conditions at different stresses due to orientation dependence of transformation strain (Šittner and Novák, 2000). Thus, we can expect that the forward transformation in polycrystalline samples induced by stress will start in the most favorably oriented grains (with the highest transformation temperature) and it will advance to other parts only with increasing loading.

In the next paragraph, we will explain the loading mode-dependence of localization of martensitic transformation in NiTi as the result of an interplay of hardening-like effects given by finite distributions of transformation strains and temperatures in a polycrystalline material and softening effects coming from the heterogeneous formation of martensite on the single-grain level and interaction of highly deformed martensitic zone with surrounding elastic austenite. The hardening during martensitic transformation was already incorporated in the original model by Sedlák et al. (2012) via the assumption of finite intervals of both forward and reverse transformations defined by transformation temperatures $M_{\mathrm{s}}, M_{\mathrm{f}}, A_{\mathrm{s}}$ and $A_{\mathrm{f}}$; the austenite-martensite interaction term is derived in the next section.

\section{Energy of austenite-martensite interaction}

Derivation of austenite-martensite interaction term on macroscopic, phenomenological level is usually based on the Eshelby's solution of elastic field of ellipsoidal inclusion in elastic matrix (Eshelbv, 1957) and the derivation of average stress in material with misfitting inclusions by Mori and Tanaka (1973).

The elastic energy per unit volume of the specimen containing elliptical inclusions with transformation strain $\varepsilon^{\text {tr }}$ reads as (Mori and Tanaka, 1973):

$$
E^{\text {int }}=-\frac{1}{2} \xi(1-\xi) \boldsymbol{\sigma}^{I \infty}: \varepsilon^{\mathrm{tr}}
$$

where $\xi$ is the volume fraction of inclusions and $\boldsymbol{\sigma}^{I \infty}$ is stress within a single inclusion existing in an infinitely extended body. Considering simple spherical inclusions, identical isotropic elasticity of both austenite and martensite and the constraint $\operatorname{tr}\left(\varepsilon^{\operatorname{tr}}\right)=0$, the energy term condensates into a simple form:

$$
E^{\text {int }}=C^{\text {int }} \xi(1-\xi)\left\|\varepsilon^{\text {tr }}\right\|^{2}
$$

where $C^{\text {int }}$ is a positive constant depending only on material elastic constants. The interaction energy given by Eq. (6) is maximal for $\xi=0.5$ and it symmetrically decreases towards $\xi=0$ and $\xi=1$ (see Fig. 1b). As it is quadratic in $\xi$, the stress would decrease linearly with increasing $\xi$ due to this interaction term, which contradicts experimental observations. The reason of the symmetry of $E^{\text {int }}$ with respect to the $\xi \leftrightarrow(1-\xi)$ is given by equality of the energy of martensite inclusions with transformation strain $\varepsilon^{\text {tr }}$ within austenite matrix and (inversely) the energy of austenite inclusions 

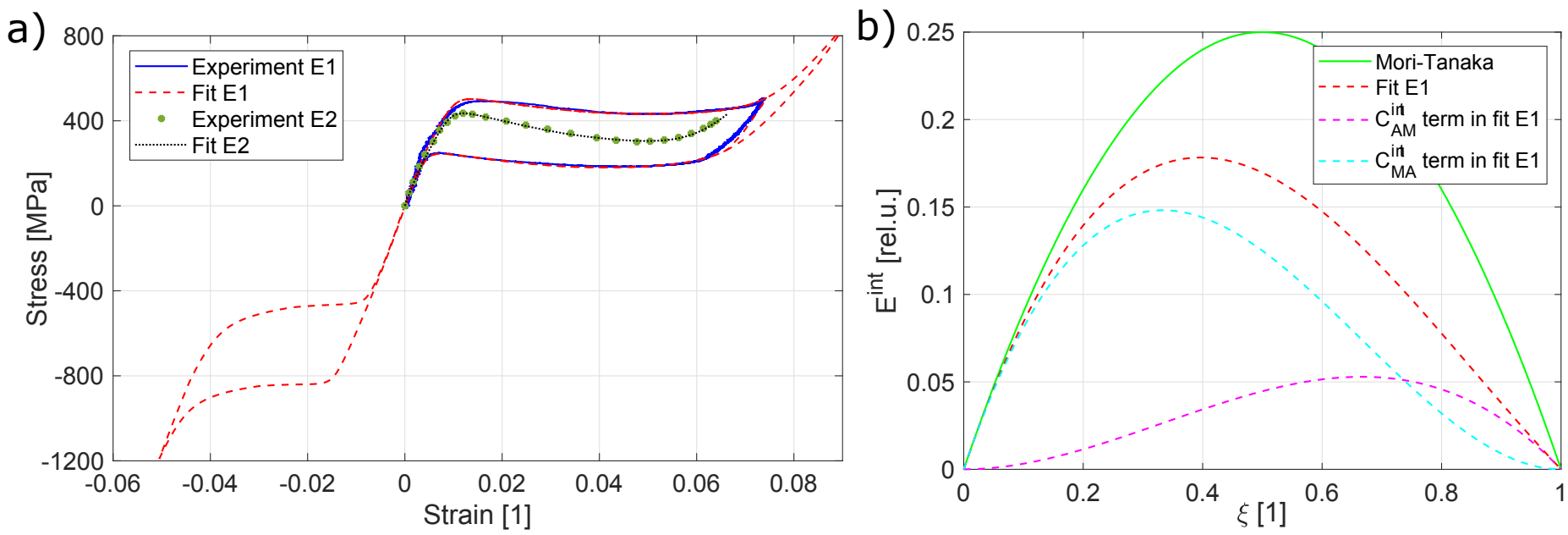

Figure 1: (a) Experimental data on strain-softening in NiTi alloys by Alarcon et al. (2017) - denoted E1 - and Hallai and Kyriakides (2013) - denoted E2 - plotted together with their best numerical fit reached by the non-local constitutive model introduced in the Section 3 . (b) Character of the evolution of the internal elastic energy $E^{\text {int }}$ with variation of the volume fraction of martensite $\xi$. For $C_{\mathrm{MA}}^{\mathrm{int}}=C_{\mathrm{AM}}^{\mathrm{int}}$ the classical Mori-Tanaka symmetric case is recovered (marked in green). The symmetry of $E^{\mathrm{int}}$ is lost in the best fit of E1 (red dashed line) since $C_{\mathrm{MA}}^{\mathrm{int}}=2.8 C_{\mathrm{AM}}^{\mathrm{int}}$, see the respective contributions of corresponding energy terms (cyan and magenta dashed lines).

without transformation strain within $\varepsilon^{\text {tr }}$-deformed martensite. According to experimental observations, this symmetry seems not fulfilled in NiTi, most likely due to nonlinear martensite behavior caused by its low reorientation stress. Indeed, if we split $E^{\text {int }}$ into two independent terms (notice that Eq. (6) is recovered for $\left.C_{\mathrm{MA}}^{\text {int }}=C_{\mathrm{AM}}^{\mathrm{int}}=C^{\mathrm{int}}\right)$ :

$$
E^{\text {int }}=C_{\mathrm{MA}}^{\text {int }} \xi(1-\xi)^{2}\left\|\varepsilon^{\mathrm{tr}}\right\|^{2}+C_{\mathrm{AM}}^{\mathrm{int}} \xi^{2}(1-\xi)\left\|\varepsilon^{\mathrm{tr}}\right\|^{2}
$$

we are able to successfully fit strain-softening material response obtained in experiments. Figure 1a) shows two uniaxial stress-strain relations extracted from dedicated experiments by Hallai and Kyriakides (2013) and Alarcon et al. (2017) together with their best fits obtained by our constitutive model. Note the exceptionally good match in the (non-linear) strain-softening stage. Figure 1b) reveals the asymmetric contributions of the first and second term on the right-hand side of the relation (17) to the total $E^{\text {int }}$ responsible for the non-linear softening in E1 (the first term dominates). The resulting $E^{\text {int }}$ in (17) can be simply considered as the energy of martensite inclusions (with transformation strain $\varepsilon^{\text {tr }}$ ) and austenite inclusions (without inelastic strain) within the average austenite-martensite matrix with inelastic strain $\xi \varepsilon^{\mathrm{tr}}$.

Finally, we can recast $E^{\text {int }}$ into a form more suitable for model regularization and implementation into FEM:

$$
E^{\mathrm{int}}=C_{\mathrm{MA}}^{\mathrm{int}} \xi\left\|\varepsilon^{\mathrm{tr}}-\left(\xi \varepsilon^{\mathrm{tr}}\right)_{\omega}\right\|^{2}+C_{\mathrm{AM}}^{\mathrm{int}}(1-\xi)\left\|\left(\xi \varepsilon^{\mathrm{tr}}\right)_{\omega}\right\|^{2} .
$$

Here, variables $\xi$ and $\varepsilon^{\text {tr }}$ describe local material properties, whereas the product arrested in $(\cdot)_{\omega}$ representing average matrix inelastic strain is obtained by averaging inelastic strain within a certain neighborhood as specified below.

\section{Global formulation and mathematical properties}

In this section, we summarize the formulation of the extended model and give the main mathematical properties. 
As already mentioned in Section 2, we assume that the specimen occupies the domain $\Omega$ in the reference configuration, on which we define all variables. We will denote by $\partial \Omega$ the boundary of the reference configuration and assume the following splitting $\partial \Omega=\Gamma_{\mathrm{D}} \cup \Gamma_{\mathrm{N}}$ with $\Gamma_{\mathrm{D}}, \Gamma_{\mathrm{N}}$ disjoint. On the part $\Gamma_{\mathrm{N}}$ the surface force $F_{\text {surf }}$ is acting on the specimen while on the part $\Gamma_{\mathrm{D}}$ Dirichlet boundary conditions for the displacement are prescribed. For simplicity, we restrict ourselves here to zero Dirichlet boundary conditions; i.e., $u(x)=0$ on $\Gamma_{\mathrm{D}}$.

Moreover, while even the elastic part of the free energy (44) is not convex in $\xi$ nor $\varepsilon^{\text {tr }}$, we shall, for mathematical considerations, rather switch variables and work with the volume fraction $\xi$ and the inelastic strain $\varepsilon^{\text {in }}$ defined in (2). In these variables then, at least the local part of the energy, is indeed convex. For the readers convenience, let us recall the definition of the energy and dissipation reformulated in the inelastic strain:

$$
\begin{aligned}
f^{T}\left(\varepsilon, \xi, \varepsilon^{\text {in }}\right) & =\frac{1}{2}\left(\varepsilon-\varepsilon^{\text {in }}\right): \mathbb{C}(\xi):\left(\varepsilon-\varepsilon^{\text {in }}\right)+\Delta s^{A M}\left(T-T_{0}\right) \xi, \\
d^{T}\left(\varepsilon^{\text {in }}, \xi, \dot{\varepsilon}^{\text {in }}, \dot{\xi}\right) & = \begin{cases}\Delta s^{A M}\left[T_{0}-M_{\mathrm{s}}+\xi\left(M_{\mathrm{s}}-M_{\mathrm{f}}\right)\right]|\dot{\xi}|+\sigma^{\mathrm{reo}}(T)\left\|\dot{\varepsilon}^{\text {in }}\right\| & \text { if } \dot{\xi} \geq 0, \\
\Delta s^{A M}\left[A_{\mathrm{f}}-T_{0}+\xi\left(A_{\mathrm{s}}-A_{\mathrm{f}}\right)\right]|\dot{\xi}|+\sigma^{\mathrm{reo}}(T)\left[\left\|\frac{\dot{\xi}}{\xi} \varepsilon^{\text {in }}\right\|+\left\|\dot{\varepsilon}^{\text {in }}-\frac{\dot{\xi}}{\xi} \varepsilon^{\text {in }}\right\|\right] & \text { if } \dot{\xi}<0 .\end{cases}
\end{aligned}
$$

Due to the non-local character of the newly added interaction energy term, we will work with the total energy given by

$$
\mathcal{E}\left(t, u, \xi, \varepsilon^{\mathrm{in}}\right):=\int_{\Omega} f^{T}\left(\varepsilon(u(x)), \xi(x), \varepsilon^{\mathrm{in}}(x)\right)+r_{\text {nonloc }}\left(\xi(x), \varepsilon^{\mathrm{in}}\right)-F_{\mathrm{vol}}(t) \cdot u \mathrm{~d} x-\int_{\Gamma_{\mathrm{N}}} F_{\mathrm{surf}}(t) \cdot u \mathrm{~d} S,
$$

where $F_{\text {vol }}$ and $F_{\text {surf }}$ is the prescribed volume force acting on the specimen. This force, as well as the surface force may depend on the time variable $t$. As we will assume that the evolution of the temperature in $T$ is a given function of time, the dependence of the total energy on the temperature is captured again through the time variable.

Recall from section 3 that energy contribution modelling localization is given by

$$
r_{\text {nonloc }}\left(\xi(x), \varepsilon^{\text {in }}(x)\right)= \begin{cases}C_{\mathrm{MA}}^{\text {int }} \frac{1}{\xi}\left\|\varepsilon^{\text {in }}(x)-\xi(x)\left(\varepsilon^{\text {in }}\right)_{\omega}\right\|^{2}+C_{\mathrm{AM}}^{\text {int }}(1-\xi)\left\|\left(\varepsilon^{\text {in }}\right)_{\omega}\right\|^{2} & \text { if } \xi>0 \\ 0 & \text { if } \xi=0\end{cases}
$$

and

$$
\left(\varepsilon^{\mathrm{in}}\right)_{\omega}(x)=\frac{1}{\int_{\Omega} \mathcal{G}_{\omega}(x-y) \mathrm{d} y} \int_{\Omega} \varepsilon^{\mathrm{in}}(y) \mathcal{G}_{\omega}(x-y) \mathrm{d} y,
$$

where $\mathcal{G}_{\omega}$ is a smooth function that models the averaging through the neighborhood. In particular, we assume that $0 \leq \mathcal{G}_{\omega}(\cdot) \leq 1$ and $\int_{\mathbb{R}^{3}} \mathcal{G}_{\omega}(x) \mathrm{d} x=1$ and that $\mathcal{G}_{\omega}$ is smooth. The particular form of $\mathcal{G}_{\omega}$ is specified in Section 5, but is not important from the mathematical point of view.

Let us also notice that we use the weighting factor $1 / \int_{\Omega} \mathcal{G}_{\omega}(x-y) \mathrm{d} y$ in front of the averaging. In most cases, this factor will be just one, but it may play a role once the point $x$ is near or on the boundary. In this situation, we apply averaging only over the available specimen.

Remark 4.1. In fact, it is unclear how exactly the averaging should be designed near the boundary of the specimen $\Omega$. Here we choose to restrict the area of averaging to $\Omega$ (as common in nonlocal models, see Peerlings et al., 2001), which essentially means that for points very near to the boundary the volume over which the averaging is performed gets smaller. Of course, from the physical point of view, this does not take into account that near the boundary the specimen experiences less geometric constrains and, e.g., the martensitic transformation may initiate easier. Nevertheless, capturing these 
effects is challenging from the modelling point of view and may have a little effect in practice. This is because the physically justified radius of averaging kernel should involve several neighboring grains forming the microstructure; in common NiTi components, this would be usually at the order of few microns, i.e. far below the reasonable mesh size.

Similarly, we define the total dissipation which, however, has only local contributions.

$$
\mathcal{D}\left(t, \xi, \varepsilon^{\text {in }}, \dot{\xi}, \dot{\varepsilon}^{\text {in }}\right):=d^{T}\left(\varepsilon^{\text {in }}(x), \xi(x), \dot{\varepsilon}^{\text {in }}(x), \dot{\xi}(x)\right) \mathrm{d} x
$$

notice that the overall dissipation depends explicitly on time which is caused by the fact that the constants in the dissipation function are dependent on the temperature, which may depend on time.

According to the generalized standard solids theory, the evolution of the specimen is given by balancing the conservative and dissipative force at all times $t \in[0, T]$, where $T$ is assumed to be the final time of the evolution. Formally, we may write that

$$
\underbrace{\partial_{\left(u, \xi, \varepsilon^{\text {in }}\right)} \mathcal{E}}_{\text {conservative force }}+\underbrace{\partial_{\left(\dot{\xi}, \dot{\varepsilon}^{\text {in }}\right)} \mathcal{D}}_{\text {dissipative force }} \ni 0 \quad \text { for all } t \in[0, T],
$$

along with the constraint that the state variables remain in the admissible space $\mathcal{Q}:=\mathcal{U} \times \mathcal{V}$ with

$$
\begin{aligned}
& \mathcal{U}:=\left\{u \in W^{1,2}\left(\Omega, \mathbb{R}^{3}\right): u=0 \text { on } \Gamma_{\mathrm{D}}\right\} \\
& \mathcal{V}:=\left\{\left(\varepsilon^{\text {in }}, \xi\right) \in W^{1,2}\left(\Omega, \mathbb{R}^{3 \times 3}\right) \times W^{1,2}(\Omega): \varepsilon^{\text {in }}\right. \text { is a traceless, symmetric matrix, } \\
&\left.\left\langle\varepsilon^{\text {in }}(x)\right\rangle \leq \xi(x) \text { for a.a. } x \in \Omega, \text { and } 0 \leq \xi(x) \leq 1 \text { for a.a. } x \in \Omega\right\},
\end{aligned}
$$

for all times $t \in[0, T]$. Here, $W^{1,2}(\Omega)$ denotes the Sobolev space of functions having square integrable derivatives.

Let us notice that (14) is indeed just a formal expression, which, due to the non-local character, needs to be formulated in an integral form. It is beyond the scope of the present work to elaborate on the mathematical properties of (14), instead we shall concentrate on time-discrete approximations of solutions of (14) that will also be computed in the numerical part.

In the spirit of (Francfort and Mielke, 2006; Mielke and Theil, 2004) we design a time-discretization of (14) via the backward Euler method. To be more specific, we introduce a partition of the timeinterval $[0, T]$ via $0=t_{0} \leq t_{1} \leq \ldots t_{N(\tau)}=\mathcal{T}$ with $\max _{i}\left(t_{i+1}-t_{i}\right) \leq \tau$ and $\tau$ being some small parameter. It is expected (even if we do not give a formal proof here) that solutions of the proposed time-discrete problems will approximate solutions of (14).

Then we call the triple $\left(u_{k}, \varepsilon_{k}^{\text {in }}, \xi_{k}\right) \in \mathcal{Q}$ a time-discrete solution of evolution at time $t_{k}$ to (14) at time-level $k=1, \ldots, N(\tau)$ if it solves

$$
\begin{aligned}
& \text { Minimize } \mathcal{E}\left(t_{k}, u, \varepsilon^{\text {in }}, \xi\right)+\mathcal{D}\left(t_{k}, \xi_{k-1}, \varepsilon_{k-1}^{\text {in }}, \xi-\xi_{k-1}, \varepsilon^{\text {in }}-\varepsilon_{k-1}^{\text {in }}\right) \\
& \text { subject to }\left(u, \varepsilon^{\text {in }}, \xi\right) \in \mathcal{Q}
\end{aligned}
$$

with $\left(u_{\tau}^{0}, \varepsilon^{\text {in }, 0}, \xi^{0}\right)=\left(u_{0}, \varepsilon_{0}^{\text {in }}, \xi_{0}\right) \in \mathcal{Q}$ defined through the initial condition.

We call the minimization problem in (TIP) the time-incremental problem. It is physically wellmotivated by the idea that upon a small change in the environment, i.e. during a small time-step, the studied system will try to find the relaxed state by minimizing the energy plus the dissipation needed to transit to the new state. In other words, the state variables describing the specimen will change if this yields a gain in the free energy larger that the dissipation.

The model proposed in this paper is well defined in the sense that (TIP) possesses a solution. This is shown in the next proposition: 
Proposition 4.2. Let $\left(\xi_{k-1} \varepsilon_{k-1}^{\text {in }}\right) \in L^{\infty}(\Omega) \times L^{\infty}\left(\Omega ; \mathbb{R}^{3 \times 3}\right)$ and that $F_{\mathrm{vol}} \in C^{0}(\Omega \times[0, T])$ as well as $F_{\text {surf }} \in C^{0}\left(\Gamma_{\mathrm{N}} \times[0, T]\right)$. Then there exists a triple $\left(u_{k}, \varepsilon_{k}^{\mathrm{in}}, \xi_{k}\right) \in \mathcal{Q}$ that solves (TIP).

In this proposition we, as is standard, denoted $L^{\infty}(\Omega)$ the space of measurable functions that are bounded almost everywhere and $C^{0}(\cdot)$ stands for the space of continuous functions.

We postpone the proof of Proposition 4.2 to the appendix and make at this points only the following remark:

Remark 4.3 (Convexity). Let us note that, thanks to the localization terms, the total energy $\mathcal{E}\left(t, u, \xi, \varepsilon^{\text {in }}\right)$ is not a convex function of its variables. This is best seen if we replaced the averaged term $\left(\varepsilon^{\text {in }}\right)_{\omega}$ by its local counterpart $\varepsilon^{\text {in }}$ and rewrite (as in Section 3 ) $\varepsilon^{\text {in }}=\xi \varepsilon^{\text {tr }}$. Then, the localization energy would correspond to a double-well potential in $\xi$, which is clearly non-convex. This feature still persists if we allow for the averaged version $\left(\varepsilon^{\text {in }}\right)_{\omega}$. However, without this averaging, it could happen that very fine spatial oscillations between austenite and martensite appear because the interface between them is allowed to be infinitely (atomically) sharp. This is prohibited by the averaging term and allows to show existence of minimizers.

Nevertheless, let us note that due to the non-convexity one can expect several difficulties in calculations: solutions to (TIP) are not necessarily unique and jumps in the temporal evolution of the variables may appear. This is related to the fact that, if the material would be completely homogeneous and there were no "localization sites", it could remain e.g. in austenite much longer than is energetically favourable and then abruptly transform at some random place (cf. Alessi and Bernardini, 2015). However, such an "indeterminacy in reponse" could be easily removed by adding an initial imperfection to the numerical model: either local variation of geometry (as in Shaw and Kvriakides, 1997a; Jiang et al., 2017a) or local variation of material properties (as in Armattoe et al., 2014; Sedmák et al., 2016).

As was already noted in (Sedlák et al., 2012), apart from the non-local term, the energy function is convex in the its variables, which can be seen by simply calculating the Hessian of $f^{T}$. Actually, one relies here on the fact that the functions $h(x, y)=x^{2} / y$ and $\tilde{h}(x, y)=x^{2} /(1-y)$ are convex, provided $0 \leq y \leq 1$. Moreover, the dissipation function is convex in the rate variables for thermodynamic consistency. This carries over to the discrete setting, as designed in (TIP).

\section{Numerical simulations}

In the following, we perform finite element simulations to illustrate the ability of the proposed constitutive model to capture the localization effect. The first simulation mimics the experimentally well-examined situation of a thin NiTi ribbon loaded in uniaxial tension (e.g. Shaw and Kyriakides, 1997b; Zhang et al., 2010; Jiang et al., 2017c; Bian et al., 2018), the other one - bending of a tube - involves also compression of the material. In both of them, we employ generic material properties with pronounced strain softening in (uniaxial) tension and strain hardening in compression, see Fig. 1 and Appendix B, and presume spatially- and temporally-constant temperature corresponding to a quasistatic superelastic loading scenario. Before presenting the results, we sketch how the constitutive model can be incorporated into the finite element method.

\subsection{Implementation into the finite element method}

We implemented the constitutive model into the finite element software package Abaqus FEA via User MATerial subroutine interface. The starting point is the time incremental problem (TIP), which can be divided into two minimization subproblems (Frost et al., 2016a). The first one corresponds to finding displacement vector $u$ for fixed internal variables and prescribed boundary conditions (and temperature), which is a standard task for any finite element software package. In case of the local 
model (2), the second subproblem shrinks into searching for optimal values of internal variables in every single material point at given strain and temperature, which is handled by the UMAT subroutine as outlined in (Sedlák et al., 2012). The numerical solution of the complete problem (TIP) is then resolved by an iterative procedure manged by Abaqus, see (Sedlák et al., 2012; Frost et al., 2016a) for details. In the nonlocal model, the evolution of internal variables in a material point inherently depends on the response of neighboring material points, hence, the individual treatment is not possible anymore.

A possible way how to implement the nonlocal integral regularization formulated in (12) was proposed by Bobinski and Tejchman $(2004$, 2005). In their approach, a virtual mesh of the computational domain is added (so that nodes of the virtual mesh coincide with those of the original mesh of the body) and nonlocal variables are treated within this mesh. As described in our previous work (Frost et al., 2018c), it is possible to complement the UMAT subroutine by subroutine UEXTERNALDB, which is activated after each increment in order to update the values of the nonlocal variable and pass them back to the UMAT. The following numerical simplifications allow to reduce computation costs (Frost et al., 2018c): (i) the integration kernel has a finite spatial coverage (cf. Peerlings et al., 2001; Jirásek and Rolshoven, 2003; Bobinski and Tejchman, 2004); (ii) in each time increment, the nonlocal variable is computed based on values of internal variables obtained in the previous converged increment (staggered computation method), (iii) only the scalar variable $\xi$ is averaged in the superelastic loading regime. Although there are more candidates for the averaging function $\mathcal{G}_{\omega}$ appearing in (12), homogeneous and isotropic ones are usually preferred in the literature mainly for practical reasons (Peerlings et al., 2001; Jirásek and Rolshoven, 2003); a typical example is the (three-dimensional) Gauss distribution defined as

$$
\mathcal{G}_{\omega}(x-y):=\frac{1}{(2 \pi)^{3 / 2} \omega^{3}} \exp \left[-\frac{\|x-y\|^{2}}{2 \omega^{2}}\right] .
$$

Let us finally note that a conceptually analogous constitutive model was successfully implemented and employed for a simulation of propagation of the martensite band front in a thin NiTi wire under tension in our previous work (Sedmák et al., 2016).

\subsection{Tension of a NiTi ribbon}

We use a geometric model of a NiTi ribbon in the form of a rectangular prism with dimensions of the base $15 \mathrm{~mm}$ and $1 \mathrm{~mm}$ and with $120 \mathrm{~mm}$ in length. The body is partitioned into a uniform mesh of 3,200 $(20 \times 1 \times 160)$ identical hexaedral (brick) elements with linear interpolation (C3D8) to reduce any directional bias. For an easy initiation of the localization pattern, a small geometric imperfection in the form of a V-shaped indent is imposed on one lateral side of the strip so that the width of the most reduced cross-section is $14.7 \mathrm{~mm}$. The indent is located $1.5 \mathrm{~mm}$ from one of the bases.

The ribbon is loaded axially by prescribing displacement boundary conditions at both bases; all other surfaces are stress free. All displacement degrees of freedom are fixed at the base closer to the indent. At the other one, the axial displacement is incrementally prescribed so that the maximum displacement-to-initial length ratio is $7.5 \%$, whereas both lateral displacements are fixed.

Figure 2 presents several snapshots of the distribution of volume fraction of martensite within the ribbon during loading. The common features of this type of localization patterns can be observed: nucleation event in the form of an inclined thin martensite band crossing the sample (1), restoring the momentum balance by nucleation of additional bands with either the opposite inclination or from the other end of the ribbon (2)-(4), propagation of bands along the sample either via movement of one inclined phase interface or via alternating between the two of them producing 


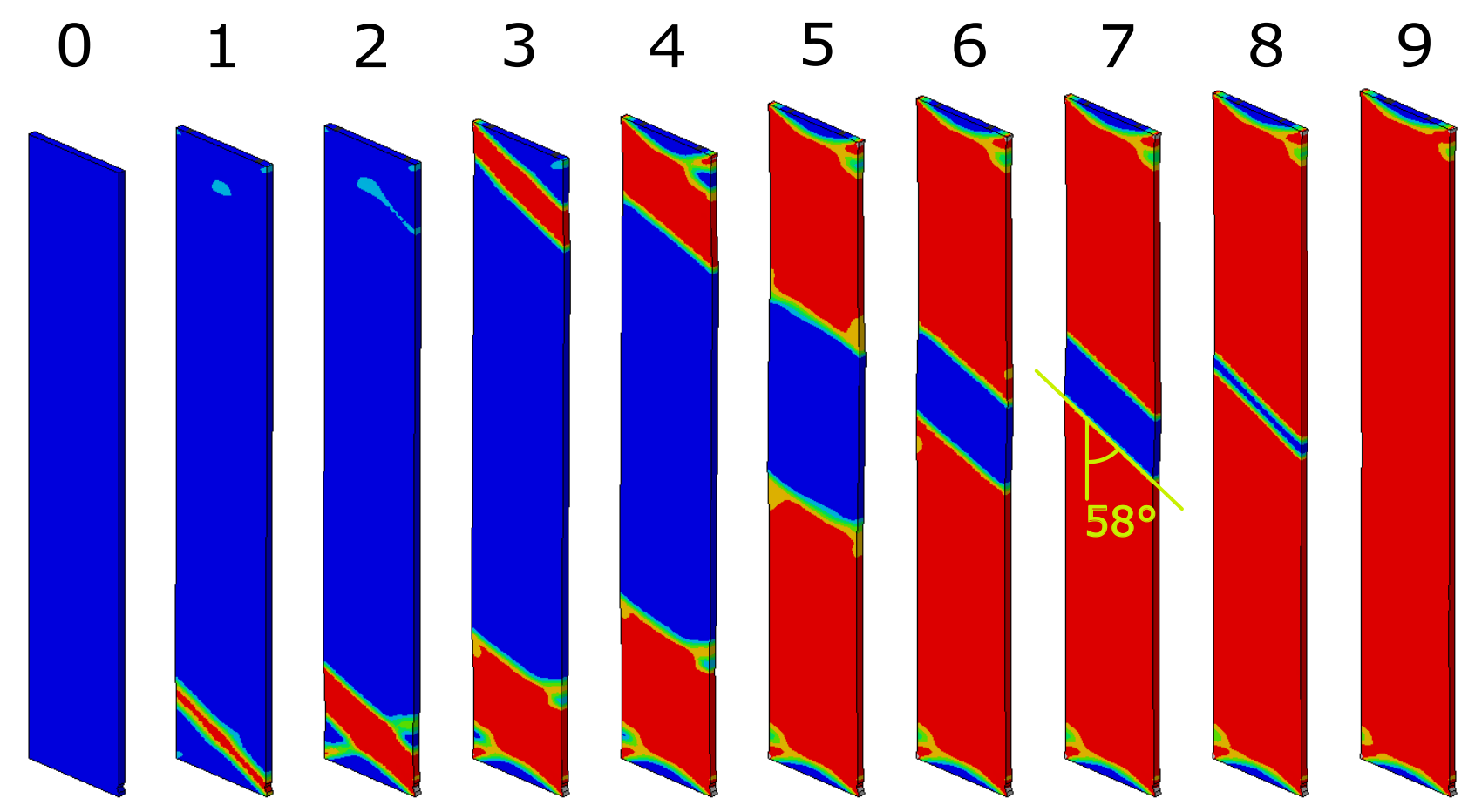

Figure 2: Snapshots from a three-dimensional simulation of the NiTi ribbon in tension with distribution of volume fraction of martensite in color (for legend see the VFM colorbar in Figure 3). The average angle of inclination of the planar martensite band front with respect to the axis of the ribbon is marked in (7).

the crisscross ("finger-like") pattern (4)-(8), and their coalescence at the final stage of loading (9). The average angle of inclination of the matensite band front (obtained from several snapshots of the simulation) is $(58 \pm 2)^{\circ}$, which is close both to experimental and modeling results obtained in the literature (cf. Shaw and Kyriakides, 1997a; Azadi et al., 2007; Grossman et al., 2010; Jiang et al., 2017c; Razaee-Haiidehi et al., 2019) and not far from the idealized theoretical two-dimensional analysis (e.g. in (Shaw and Kyriakides, 1997a)) giving the value $54.74^{\circ}$.

\subsection{Bending of a NiTi tube}

We consider an ideal tube of (outer) diameter $3.5 \mathrm{~mm}$, wall thickness $0.25 \mathrm{~mm}$ and length $25 \mathrm{~mm}$ partitioned into a uniform mesh of hexaedral elements (C3D8R) with four elements through the thickness, 96 along the circumference and 250 along the length (96,000 elements in total), i.e. full 3D geometry is modelled. Displacement boundary conditions are imposed on both bases (annuli) of the tube, remaining surfaces of the tube are stress free. Utilizing the ${ }^{*}$ COUPLING $*$ KINETIC feature of the Abaqus CAE software, mutually inverse rotation of both annular surfaces around an axis perpendicular to the tube axis is prescribed $\left( \pm 25^{\circ}\right)$, which leads to the desired bending of the whole tube, and rigid body motion is excluded.

The simulation is motivated by dedicated experiments published in (Bechle and Kyriakides, 2014; Reedlunn et al., 2014; Watkins et al., 2018; Jiang et al., 2017a). Just as in the previous section, we focus on demonstrating the capability of the proposed model to capture the key features of the behavior rather than attempting to reproduce the experiments quantitatively. However, with respect to the diameter to thickness ratio and to the boundary conditions, we are closer to the work of Watkins et al. (2018).

In Fig. 3 we present both strain and phase distributions to emphasize the different values of maximum transformation strains linked with full martensite in tension and compression, cf. Fig. 1a). The $\varepsilon_{z z}$ strain is the diagonal component of the total strain tensor in the direction of the symmetry 


\section{$\varepsilon_{\mathrm{zz}}$}

\section{0}

1

2

3
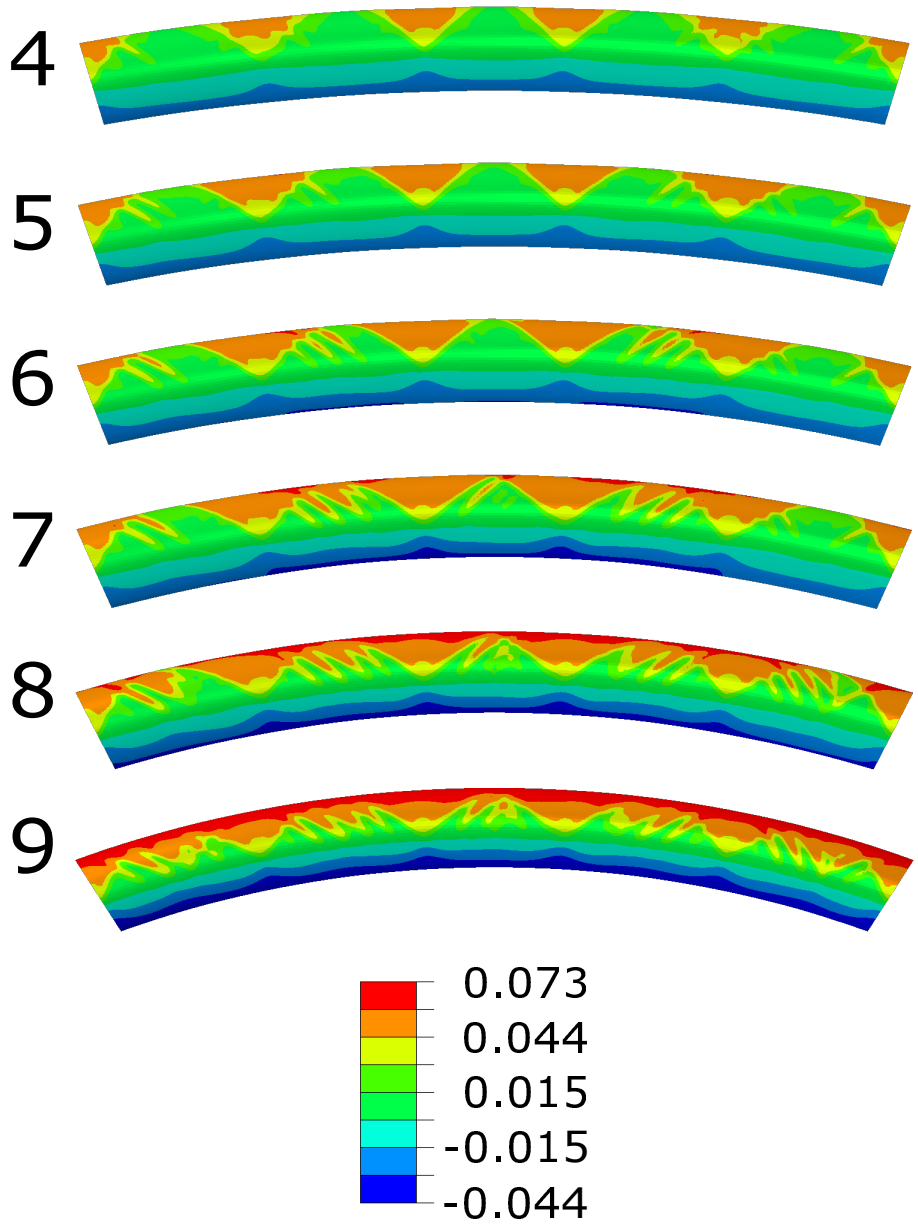

\section{VFM}
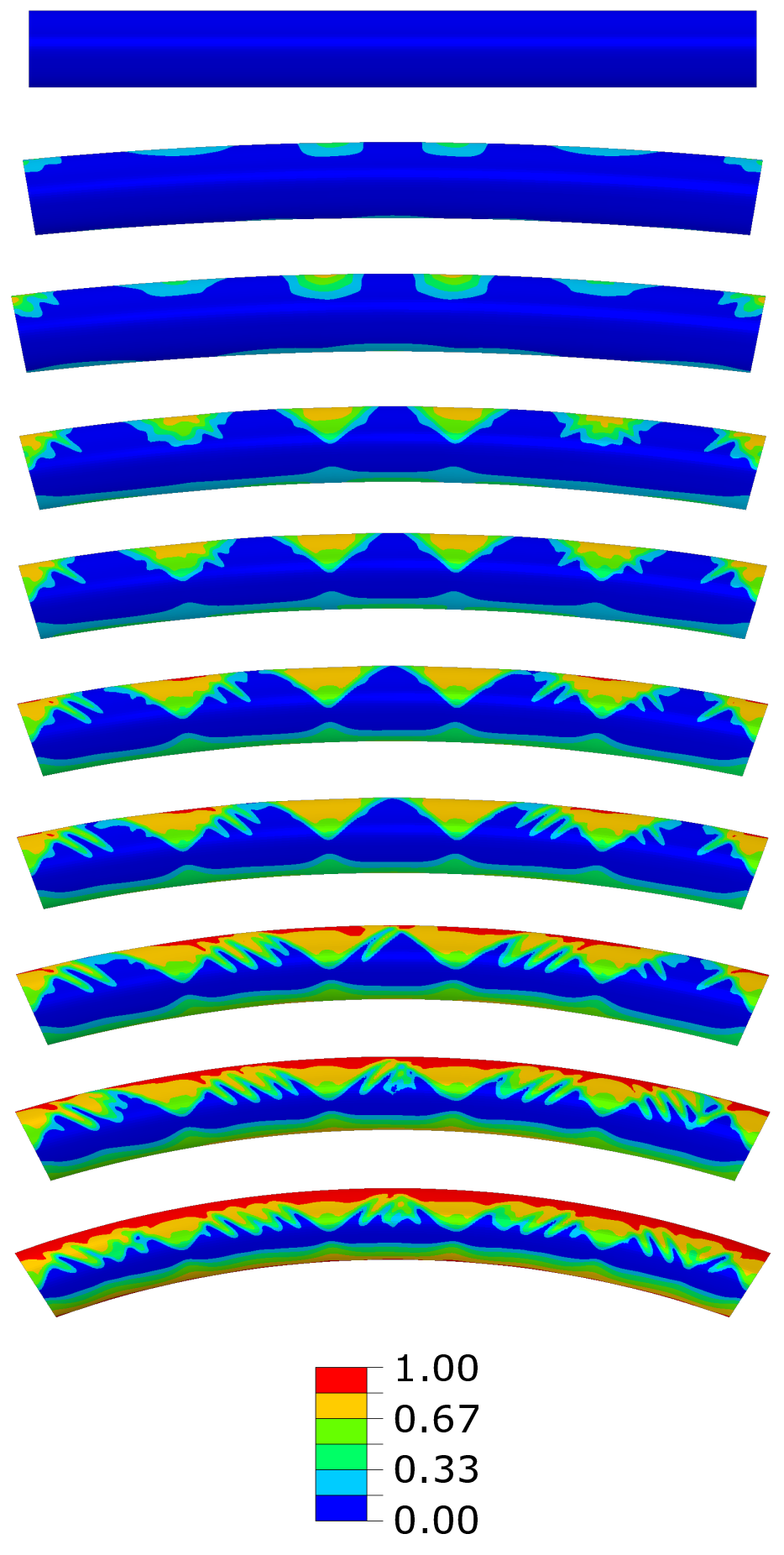

Figure 3: Snapshots from a three-dimensional simulation of the NiTi tube in bending. The distribution of the axial component of strain on the left, the distribution of the volume fraction of martensite on the right. 
axis of the tube in the reference configuration. Again, one can observe several common features of the localization patterning: appearance of inclined finger-like structures of high strain (4), (5), their growth and multiplication (6),(7), and their crisscrossing and coalescence into wedge-shaped regions (8),(9) covering most of the part of surface undergoing predominantly tensile straining. The shift of the neutral axis effectively increasing this part of the surface can be observed in accordance with experimental observations in (Watkins et al., 2018; Jiang et al., 2017a). The tension-compression asymmetry embodied in the constitutive law leads to much lower (absolute) values of strain on the lower part of the tube, and, foremost, to the homogeneous distribution of strain (and VFM) there. For comparison, we performed an identical simulation except for a modified constitutive law incorporating strain-softening also in compression. In that case, the wedge-like localization pattern was observed (not presented here) also on the lower part of the tube (where compression loading dominates) (also cf. Jiang et al., 2017a).

\section{Discussion and Conclusions}

We extended a well-established constitutive model tailored for NiTi-based shape memory alloys by an interaction energy term allowing to capture the localization effects. The summary of experimental observations in Section 3 provided hints for searching a suitable form of such an energy term within the classical Mori-Tanaka approach. The key features of the final form given by Eq. (77) are: (i) it is a sum of two independent terms, (ii) both terms are non-convex in $\xi$, (iii) both terms exhibit quadratic dependence on $\left\|\varepsilon^{\mathrm{tr}}\right\|$.

(i) Thanks to two independent constants appearing in the interaction energy, we gained more freedom to adjust the strain-softening constitutive law to available experimental measurements, as illustrated in Fig. 1; particularly, we were not limited to the solely linear decrease of stress with strain.

(ii) Adopting the localization contribution energy $E^{\text {int }}$ means that the overall energy becomes nonconvex. This is, of course, a desired effect because in this way it prefers the pure states of austenite as well as martensite, hence the material transforms in a localized rather than a homogeneous way. Nevertheless, non-convex energy contributions always present possible difficulties from the mathematical as well as the computational point of view.

First, it may happen that, because of the non-convexity, solutions to the time-incremental problems (TIP) do not even exist. This is typically caused by the appearance spatial oscillations that tend to be infinitely fine. Indeed, it could happen that the material would develop "infinitely thin" stripes of martensite as well as austenite, which would effectively prohibit the existence of solutions. In numerical implementations, the width of such oscillations would be given just by the mesh-size; this holds true even in the case one interface would develop. We exclude such pathological behavior by averaging in some variables in the non-local term, which introduces a final width of the austenite-martensite interface.

While introducing non-locality allows us to exclude possible undesired effects, this reflects in more costly calculations. Nonetheless, in many situations, it is justified to replace the non-local averaged quantities by local ones. Particularly, in the three-dimensional setting, for a large class of loading regimes, an infinitely fine austenite-martensite interface cannot be formed just by geometric reasons leading naturally to the presence of a phase gradient region between the two phases 3

\footnotetext{
${ }^{3}$ Hence, in practice, it may be enough to do all calculations using just the local variables and checking at the same time the size of the gradient of the volume fraction of martensite. If this does not get too large, it is justified to perform the calculations in local variables only, which reduces the computational cost.
} 
Finally, let us notice that independently of whether we use the averaging kernels or not, nonconvexity of the problem is connected with the appearance of multiple local minimizers in (TIP). As, in numerical implementations, we can always find only local minimizers, this may lead to non-uniqueness of the results. A typical situation is that in calculations the austenitemartensite transformation happens too late. To avoid this, an effective strategy is to add e.g. stress concentrators into the model.

Let us note that the above observations are also valid if we did not solve the minimization problem (TIP) but instead the associated variational inequalities.

(iii) The factor $\left\|\varepsilon^{\operatorname{tr}}\right\|^{2}$ in the interaction energy brings the loading-mode dependency into the model: when transformation strain for a particular loading direction is large, the interaction term dominates over the loading-mode insensitive "transformation hardening" defined in the model by the difference of transformation temperatures $\left(M_{s}-M_{f}\right)$ and $\left(A_{s}-A_{f}\right)$. If transformation strain in tension is almost twice the transformation strain in compression (as suggested by experiments), the interaction energy is almost four times larger in tension than in compression; this results in strain-softening response in tension and hardening-like in compression.

This observation emphasizes the need for correct description of the so-called transformation strain surface (i.e. the boundary of the set of all available transformation strain tensors), which is defined by a 1-homogeneous convex function in our model, see the constraint in (3) and Appendix B. Our particular form of the transformation strain surface allows to capture tension/compression asymmetry of the maximum transformation strain (by involving the third invariant of the strain tensor), but does not include possible material anisotropy. The question of a suitable form of the transformation strain surface appropriate for simulations of $\mathrm{NiTi}$ components still remains open (Sedlák and Frost, 2018), and several experimental works suggest different forms involving also material anisotropy given by usually strong $\langle 111\rangle$ texture in NiTi drawn components (Reedlunn et al., 2012; Bechle and Kyriakides, 2016).

Involving effects of material texture and anisotropy by redefining transformation strain surface could be done straightforwardly in our model as we only assumed that the surface is described by a 1-homogeneous convex function. However, it is questionable whether all experimentally observed effects of material anisotropy on localization of martensitic transformation can be covered solely by a proper description of the transformation strain surface. For example, microstructure observations of martensitic transformation on NiTi twisted tube by Peng et al. (2008) revealed that martensite appears in the form of almost parallel lamellae with inclination about $26.5^{\circ}$ from the axial direction of the tube. Such a strong preferential orientation of austenite-martensite habit planes can be explained by $\langle 111\rangle$ material texture. By calculating all possible habit plane orientations - 24 Type II habit plane variants from (Matsumoto et al., 1987) were considered - using the mathematical theory of martensitic microstructures by Ball and James (1987), it can be shown that there are several possible habit planes nearly parallel to the $[111]_{\mathrm{A}}$ direction (there are at least nine different habit planes inclined less than $10^{\circ}$ w.r.t. $[111]_{\mathrm{A}}$ direction), while there are no habit planes nearly perpendicular to this direction (the normal to the least favourable one is inclined just in an angle of $16.5^{\circ}$ w.r.t. $[111]_{\mathrm{A}}$ ). Among the nine habit planes nearly parallel to $[111]_{\mathrm{A}}$, three possible orientations correspond to remarkably large resolved shear strains. Hence, it is plausible that the first nuclei of stress-induced martensite can be encapsulated by such habit planes under the applied twisting. Such a strong orientation of forming microstructure could alter both expression of the interaction energy (derived in the Section 3 with the assumption of spherical inclusions) but also the way of calculation of nonlocal variables: the isotropic integration kernel in Eq. (17) could be replaced by an anisotropic one to reflect directional dependence of interaction between inclusions. We plan to address these issues in future work. 
Finally, in addition to the above discussed key features of the definition (17), its motivation stemming from the Mori-Tanaka method also provided a hint for the physically plausible regularization of the non-convex model expressed by (8). Then, we could proceed by performing its basic mathematical analysis, which provided a sound basis for a numerical implementation of the time-discretized problem TIP into FEM excluding pathological behavior in simulations.

The simulation of a NiTi tube subjected to bending showed that the model is able to capture the difference between loading in tension - where characteristic patterns of localized deformation appeared - and compression - where homogeneous deformation persists. In our previous work (Frost et al., 2018b), the model was also employed in a study on bending of a NiTi wire structure. Thanks to direct comparison of simulations with x-ray microdiffraction data, it was confirmed that localization strongly affects the mechanical response also in such a case.

The computed localization patterns on the bent tube as well as those on the NiTi ribbon under tension exhibited some features commonly observed in experiments. As pointed out by many authors (e.g. Šittner et al., 2005; Grossman et al., 2010; Xiao et al., 2017; Zhang and He, 2018), particular geometrical forms and propagation modes of the localization bands - even for the specific type of sample geometry and loading mode - strongly depend on the material (composition, processing), dimensions of the sample, and boundary conditions 4 Thus, the presented FE model also provides a powerful tool for further exploration of these issues.

\section{Acknowledgement}

We acknowledge the financial support of the Czech Science Foundation via project No. GA18$03834 \mathrm{~S}$ and of the Ministry of Education, Youth and Sports via project

No. CZ.02.1.01/0.0/0.0/16_013/0001794 (European Spallation Source - participation of the Czech Republic - OP) funded within the Operational Programme Research, Development and Education. We would like to thank Dr. E. Alarcon for providing us the experimental data used in Fig. 1a).

\section{Appendix A. Mathematical analysis}

In this section, we prove Proposition 4.2 by the direct method of calculus of variations (cf. e.g. (Dacorogna, 2008)). To do so, we rely on the convexity of the minimized function in all term except the localization one, in the latter we rely on the used averaging. In fact, we first need to realize that the localization contribution to the energy is continuous, i.e. that $r_{\text {nonloc }}\left(\xi, \varepsilon^{\text {in }}\right)$ is a continuous function on $[0,1] \times \mathbb{R}^{3 \times 3}$. The only thing we have to verify is that

$$
\lim _{\xi \rightarrow 0} \frac{\left\|\varepsilon^{\text {in }}-\xi\left(\varepsilon^{\text {in }}\right)_{\omega}\right\|^{2}}{\xi}=0 .
$$

We use that $\langle\cdot\rangle$ is an equivalent norm on $\mathbb{R}^{3 \times 3}$ meaning that there exists a constant $\mathrm{c}$ such that $\|\varepsilon\| \leq c\langle\varepsilon\rangle$ for some constant $c$ and all $\varepsilon \in \mathbb{R}^{3 \times 3}$. Owing to this

$$
0 \leq \frac{\left\|\varepsilon^{\text {in }}-\xi\left(\varepsilon^{\text {in }}\right)_{\omega}\right\|^{2}}{\xi} \leq c \frac{\left\langle\varepsilon^{\text {in }}-\xi\left(\varepsilon^{\text {in }}\right)_{\omega}\right\rangle^{2}}{\xi} \leq c \frac{\xi^{2}+\xi^{2}\left\langle\left(\varepsilon^{\text {in }}\right)_{\omega}\right\rangle^{2}}{\xi} \stackrel{\xi \rightarrow 0}{\longrightarrow} 0
$$

where we also used that $\left\langle\varepsilon^{\text {in }}\right\rangle \leq \xi$. Furthermore, $r_{\text {nonloc }}\left(\xi, \varepsilon^{\text {in }}\right)$ is convex function in $\left.\varepsilon^{\text {in }}, \xi\right)$, if we regard $\left.\varepsilon^{\text {in }}\right)_{\omega}$ as a fixed independent variable. This follows from the positive definiteness of the Hessian and is related to the fact that $\frac{x^{2}}{y}$ is a convex function for $x, y>0$.

\footnotetext{
${ }^{4}$ And, beyond the quasistatic approximation, they also depend on the deformation rate (Zhang et al., 2010).
} 
Let us now turn to proving existence of minimizers to (TIP). We find a minimizing sequence $\left[u^{j},\left[\varepsilon^{\mathrm{in}}\right]^{j}, \xi^{j}\right)_{j \in \mathbb{N}} \subset \mathcal{Q}$ so that (for $j \rightarrow \infty$ )

$$
\mathcal{E}\left(t_{k}, u^{j},\left[\varepsilon^{\mathrm{in}}\right]^{j}, \xi^{j}\right)+\mathcal{D}\left(t_{k}, \xi_{k-1}, \varepsilon_{k-1}^{\mathrm{in}}, \xi^{j}-\xi_{k-1},\left[\varepsilon^{\mathrm{in}}\right]^{j}-\varepsilon_{k-1}^{\mathrm{in}}\right) \rightarrow \text { inf. }
$$

Due to the quadratic growth of the energy in the elastic part as well as due to the bounds imposed in $\mathcal{Q}$, we can assume that (at least for a subsequence denoted by the same indices) we have the following convergence results for $j \rightarrow \infty$ :

$$
\begin{aligned}
u^{j} \rightarrow u_{k} & \text { in } W^{1,2}\left(\Omega ; \mathbb{R}^{3}\right), \\
{\left[\varepsilon^{\text {in }}\right]^{j} \stackrel{*}{\rightarrow} \varepsilon_{k}^{\text {in }} } & \text { in } L^{\infty}\left(\Omega ; \mathbb{R}^{3 \times 3}\right), \\
\xi^{j} \stackrel{*}{\rightarrow} \xi_{k} & \text { in } L^{\infty}(\Omega) .
\end{aligned}
$$

We now show that $\left(u_{k}, \varepsilon_{k}^{\text {in }}, \xi_{k},\right) \in W^{1,2}\left(\Omega ; \mathbb{R}^{3}\right) \times L^{\infty}\left(\Omega ; \mathbb{R}^{3 \times 3}\right) \times L^{\infty}(\Omega)$ belongs to $\mathcal{Q}$ is a solution of (TIP). Due to the convexity of the constraints, we know that

$$
\left\langle\varepsilon_{k}^{\text {in }}(x)\right\rangle \leq \xi_{k}(x), \quad \text { and } \quad 0 \leq \xi_{k}(x) \leq 1, \quad \text { a.e. on } \Omega .
$$

Moreover, due to the convexity of the local part of the energy as well as the dissipation (in the rate variable) we see that

$$
\begin{gathered}
\int_{\Omega} f^{T}\left(\varepsilon\left(u_{k}(x)\right), \xi_{k}(x), \varepsilon_{k}^{\text {in }}(x)\right)-F_{\text {vol }}\left(t_{k}\right) \cdot u_{k}+d^{T_{k}}\left(\xi_{k-1}, \varepsilon_{k-1}^{\text {in }}, \xi_{k}-\xi_{k-1}, \varepsilon_{k}^{\text {in }}-\varepsilon_{k-1}^{\text {in }}\right) \mathrm{d} x \\
-\int_{\Gamma_{\mathrm{N}}} F_{\text {surf }}\left(t_{k}\right) \cdot u_{k} \mathrm{~d} S \\
\leq \liminf _{j \rightarrow \infty} f^{T}\left(\varepsilon\left(u^{j}(x)\right), \xi^{j}(x),\left[\varepsilon^{\mathrm{in}}\right]^{j}(x)\right)-F_{\mathrm{vol}}\left(t_{k}\right) \cdot u^{j}+d^{T_{k}}\left(\xi_{k-1}, \varepsilon_{k-1}^{\mathrm{in}}, \xi^{j}-\xi_{k-1},\left[\varepsilon^{\mathrm{in}}\right]^{j}-\varepsilon_{k-1}^{\mathrm{in}}\right) \mathrm{d} x \\
\quad-\int_{\Gamma_{\mathrm{N}}} F_{\mathrm{surf}}\left(t_{k}\right) \cdot u^{j} \mathrm{~d} S
\end{gathered}
$$

So we only need to look at the nonlocal part. To this end, we realize that, for any fixed $\omega>0$, we have that, for all $x \in \Omega$

$$
\left(\left[\varepsilon^{\mathrm{in}}\right]^{j}\right)_{\omega}(x)=\int_{\Omega}\left[\varepsilon^{\mathrm{in}}\right]^{j}(y) \mathcal{G}_{\omega}(x-y) \mathrm{d} y \rightarrow \int_{\Omega} \varepsilon_{k}^{\mathrm{in}}(y) \mathcal{G}_{\omega}(x-y) \mathrm{d} y=\left(\varepsilon_{k}^{\mathrm{in}}\right)_{\omega}(x),
$$

so that $\left(\left[\varepsilon^{\text {in }}\right]^{j}\right)_{\omega} \rightarrow\left(\varepsilon^{\text {in }}\right)_{\omega}$ pointwise in $\Omega$. Furthermore, since $\left(\left[\varepsilon^{\text {in }}\right]^{j}\right)_{\omega}(x)$ is uniformly bounded, this extends to strong convergence in $L^{p}\left(\Omega ; \mathbb{R}^{3 \times 3}\right)$ for all $p \in[1, \infty)$. For the first term in the localization energy we use the rewriting

$$
\begin{aligned}
& \frac{\left\|\left[\varepsilon^{\mathrm{in}}\right]^{j}-\xi^{j}\left(\left[\varepsilon^{\mathrm{in}}\right]^{j}\right)_{\omega}\right\|^{2}}{\xi^{j}}=\frac{\left\|\left[\varepsilon^{\mathrm{in}}\right]^{j}-\xi^{j}\left(\left[\varepsilon^{\mathrm{in}}\right]^{j}\right)_{\omega}-\xi^{j}\left(\varepsilon_{k}^{\mathrm{in}}\right)_{\omega}+\xi^{j}\left(\varepsilon_{k}^{\mathrm{in}}\right)_{\omega}\right\|^{2}}{\xi^{j}} \\
& \quad=\frac{\left\|\left[\varepsilon^{\mathrm{in}}\right]^{j}-\xi^{j}\left(\varepsilon_{k}^{\mathrm{in}}\right)_{\omega}\right\|^{2}}{\xi^{j}}+2\left(\left[\varepsilon^{\mathrm{in}}\right]^{j}-\xi^{j}\left(\varepsilon_{k}^{\mathrm{in}}\right)_{\omega}\right) \cdot\left(\left(\left[\varepsilon^{\mathrm{in}}\right]^{j}\right)_{\omega}-\left(\varepsilon_{k}^{\mathrm{in}}\right)_{\omega}\right)+\xi^{j}\left\|\left(\left[\varepsilon^{\mathrm{in}}\right]^{j}\right)_{\omega}-\left(\varepsilon_{k}^{\mathrm{in}}\right)_{\omega}\right\|^{2},
\end{aligned}
$$

we see that the first term in the second line is convex in $\left(\xi_{j},\left[\varepsilon^{\mathrm{in}}\right]^{j}\right)$, so that its integral is weakly lower semicontinuous and the integral of the latter two converges to 0 as $j \rightarrow \infty$. For the second term in the localization energy, we only need to look at

$$
\xi^{j}\left\|\left(\left[\varepsilon^{\mathrm{in}}\right]^{j}\right)_{\omega}\right\|^{2}=\xi^{j}\left\|\left(\varepsilon_{k}^{\mathrm{in}}\right)_{\omega}\right\|^{2}-2 \xi^{j}\left(\varepsilon_{k}^{\mathrm{in}}\right)_{\omega} \cdot\left(\left(\left[\varepsilon^{\mathrm{in}}\right]^{j}\right)_{\omega}-\left(\varepsilon_{k}^{\mathrm{in}}\right)_{\omega}\right)+\xi^{j}\left\|\left(\left[\varepsilon^{\mathrm{in}}\right]^{j}\right)_{\omega}-\left(\varepsilon_{k}^{\mathrm{in}}\right)_{\omega}\right\|^{2},
$$

where again the the first term on the right hand side is even linear in $\xi^{j}$ while the other two tend to zero as $j \rightarrow \infty$.

Combining the arguments above implies that $\left(u_{k}, \varepsilon_{k}^{\text {in }}, \xi_{k}\right) \in W^{1,2}\left(\Omega ; \mathbb{R}^{3}\right) \times L^{\infty}\left(\Omega ; \mathbb{R}^{3 \times 3}\right) \times L^{\infty}(\Omega)$ is a solution of (TIP) 
Table B.1: Table of material parameters used in computations.

\begin{tabular}{|c|c|c|c|}
\hline Parameter & Value & Unit & Brief description \\
\hline $\bar{K}$ & 148 & {$[\mathrm{GPa}]$} & Bulk modulus common to both phases. \\
\hline$G^{\mathrm{A}}, G^{\mathrm{M}}$ & 25,15 & {$[\mathrm{GPa}]$} & Shear moduli of austenite and martensite. \\
\hline$k$ & 0.072 & {$[1]$} & Maximum transformation strain in tension. \\
\hline$a$ & 0.99 & {$[1]$} & Tension-compression asymmetry parameter. \\
\hline$A_{\mathrm{s}}, A_{\mathrm{f}}$ & $-30,-18$ & {$\left[{ }^{\circ} \mathrm{C}\right]$} & $\begin{array}{l}\text { Martensite to austenite transformation tempera- } \\
\text { tures. }\end{array}$ \\
\hline$M_{\mathrm{s}}, M_{\mathrm{f}}$ & $-37,-47$ & {$\left[{ }^{\circ} \mathrm{C}\right]$} & $\begin{array}{l}\text { Austenite to martensite transformation tempera- } \\
\text { tures. }\end{array}$ \\
\hline$T_{0}$ & -35 & {$\left[{ }^{\circ} \mathrm{C}\right]$} & Equilibrium austenite-martensite temperature. \\
\hline$\sigma^{\text {reo }}$ & 85 & {$[\mathrm{MPa}]$} & Martensite reorientation stress. \\
\hline$\Delta s^{\mathrm{AM}}$ & 0.36 & {$\left[\mathrm{MPa} /{ }^{\circ} \mathrm{C}\right]$} & $\begin{array}{l}\text { Difference between specific entropies of martensite } \\
\text { and austenite. }\end{array}$ \\
\hline$C_{\mathrm{MA}}^{\mathrm{int}}$ & 80 & {$[\mathrm{MPa}]$} & Parameter of the interaction energy in Eq. (8). \\
\hline$C_{\mathrm{AM}}^{\mathrm{int}}$ & 29 & {$[\mathrm{MPa}]$} & Parameter of the interaction energy in Eq. (88). \\
\hline
\end{tabular}

\section{Appendix B. Material parameters}

The material parameters obtained as the best fit of experimental data E1 (Alarcon et al., 2017) in Fig. 1 are summarized in Tab. B.1.

The particular form of the function confining the transformation strain $\langle\cdot\rangle$ from constraints (3) and defining the transformation strain surface is (cf. Sedlák et al., 2012; Sedlák and Frost, 2018):

$$
\left\langle\varepsilon^{\operatorname{tr}}\right\rangle=\frac{I_{2}\left(\varepsilon^{\operatorname{tr}}\right)}{k} \frac{\cos \left(\frac{1}{3} \arccos \left(1-a\left(I_{3}\left(\varepsilon^{\operatorname{tr}}\right)+1\right)\right)\right)}{\cos \left(\frac{1}{3} \arccos (1-2 a)\right)}
$$

where

$$
I_{2}(\boldsymbol{x}):=\sqrt{\frac{2}{3} x_{i j} x_{i j}}, \quad I_{3}(\boldsymbol{x}):=4 \frac{\operatorname{det}(\boldsymbol{x})}{\left(I_{2}(\boldsymbol{x})\right)^{3}} .
$$

\section{References}

\section{References}

Ahmadian, H., Ardakani, S.H., Mohammadi, S., 2015. Strain-rate sensitivity of unstable localized phase transformation phenomenon in shape memory alloys using a non-local model. Int. J. Solids Struct. 63, 167-183.

Alarcon, E., Heller, L., Chirani, S., Šittner, P., Kopeček, J., Saint-Sulpice, L., Calloch, S., 2017. Fatigue performance of superelastic NiTi near stress-induced martensitic transformation. Int. J. Fatigue 95, 76-89.

Alessi, R., Bernardini, D., 2015. Analysis of localization phenomena in shape memory alloys bars by a variational approach. Int. J. Solids Struct. 73-74, 113-133. 
Armattoe, K., Haboussi, M., Ben Zineb, T., 2014. A 2D finite element based on a nonlocal constitutive model describing localization and propagation of phase transformation in shape memory alloy thin structures. Int. J. Solids Struct. 51, 1208-1220.

Auricchio, F., Bonetti, E., Scalet, G., Ubertini, F., 2014. Theoretical and numerical modeling of shape memory alloys accounting for multiple phase transformations and martensite reorientation. Int. J. Plast. 59, 30-54.

Azadi, B., Rajapakse, R.D., Maijer, D.M., 2007. Multi-dimensional constitutive modeling of SMA during unstable pseudoelastic behavior. Int. J. Solids Struct. 44, 6473-6490.

Badnava, H., Kadkhodaei, M., Mashayekhi, M., 2014. A non-local implicit gradient-enhanced model for unstable behaviors of pseudoelastic shape memory alloys in tensile loading. Int. J. Solids Struct. $51,4015-4025$.

Ball, J., James, R., 1987. Fine phase mixtures as minimizers of energy. Arch. Ration. Mech. An. 100, 13-52. doi:10.1007/BF00281246.

Bažant, Z., Jirásek, M., 2002. Nonlocal integral formulations of plasticity and damage: Survey of progress. J. Eng. Mech. 128, 1119-1149.

Bechle, N., Kyriakides, S., 2014. Localization in NiTi tubes under bending. Int. J. Solids Struct. 51, 967-980.

Bechle, N., Kyriakides, S., 2016. Evolution of phase transformation fronts and associated thermal effects in a NiTi tube under a biaxial stress state. Extrem Mech. Letters 8, 55-63.

Bian, X., Saleha, A., Pereloma, E., Davies, C., Gazder, A., 2018. A digital image correlation study of a NiTi alloy subjected to monotonic uniaxial and cyclic loading-unloading in tension. Materials Sci. Eng. A 726, 102-112.

Bobinski, J., Tejchman, J., 2004. Numerical simulations of localization of deformation in quasi-brittle materials within non-local softening plasticity. Comp. Concrete 1, 433-455.

Bobinski, J., Tejchman, J., 2005. Modelling of concrete behaviour with a non-local continuum damage approach. Arch. Hydro-Eng. Environ. Mech. 52, 243-263.

Chatziathanasiou, D., Chemisky, Y., Chatzigeorgiou, G., Meragni, F., 2016. Modeling of coupled phase transformation and reorientation in shape memory alloys under non-proportional thermomechanical loading. Int. J. Plast. 82, 192-224.

Chemisky, Y., Duval, A., Patoor, E., Ben Zineb, T., 2011. Constitutive model for shape memory alloys including phase transformation, martensitic reorientation and twins accommodation. Mech. Mater. 43, 361-376.

Cisse, C., Zaki, W., Ben Zineb, T., 2016a. A review of constitutive models and modeling techniques for shape memory alloys. Int. J. Plast. 76, 244-284.

Cisse, C., Zaki, W., Ben Zineb, T., 2016b. A review of modeling techniques for advanced effects in shape memory alloy behavior. Smart Mater. Struct. 25, 103001.

Dacorogna, B., 2008. Direct Methods in the Calculus of Variations. volume 78 of Applied Mathematical Sciences. 2nd ed., Springer-Verlag, New York. 
Duval, A., Haboussi, M., Ben Zineb, T., 2011. Modelling of localization and propagation of phase transformation in superelastic SMA by a gradient nonlocal approach. Int. J. Solids Struct. 48, 1879-1893.

Elibol, C., Wagner, M.X., 2015. Investigation of the stress-induced martensitic transformation in pseudoelastic NiTi under uniaxial tension, compression and compression-shear. Mater. Sci. Eng. A $621,76-81$.

Eshelby, J., 1957. The determination of the elastic field of an ellipsoidal inclusion, and related problems. Proc. R. Soc. London, Ser. A 241, 376-396.

Francfort, G., Mielke, A., 2006. Existence results for a class of rate-independent material models with nonconvex elastic energies. J. Reine Angew. Math. 595, 55-91.

Frost, M., Benešová, B., Sedlák, P., 2016a. A microscopically motivated constitutive model for shape memory alloys: formulation, analysis and computations. Math. Mech. Solids 21, 358-382.

Frost, M., Sedlák, P., Ben Zineb, T., 2018a. Experimental observations and modeling of localization in superelastic NiTi polycrystalline alloys: State of the art. Acta Phys. Pol. A 134, 847-.

Frost, M., Sedlák, P., Heller, L., Kadeřávek, L., Šittner, P., 2018b. Experimental and computational study on phase transformations in superelastic NiTi snake-like spring. Smart Mater. Struct. 27, 095005 .

Frost, M., Sedlák, P., Kadeřávek, L., Heller, L., Šittner, P., 2016b. Modeling of mechanical response of NiTi shape memory alloy subjected to combined thermal and non-proportional mechanical loading: a case study on helical spring actuator. J. Intel. Mat. Syst. Str. 27, 1927-1938.

Frost, M., Sedlák, P., Kruisová, A., Landa, M., 2014. Simulations of self-expanding braided stent using macroscopic model of NiTi shape memory alloys covering R-phase. J. Mater. Eng. Perform. 23, 2584-2590.

Frost, M., Sedlák, P., Sedmák, P., Heller, L., Šittner, P., 2018c. SMA constitutive modeling backed up by 3D-XRD experiments: Transformation front in stretched NiTi wire. Shap. Mem. Superelasticity $4,411-416$.

Grossman, C., Schaefer, A., Wagner, M.F.X., 2010. A finite element study on localized deformation and functional fatigue in pseudoelastic NiTi strips. Mater. Sci. Eng. A 527, 1172-1178.

Gu, X., Zaki, W., Morin, C., Moumni, Z., Zhang, W., 2015. Time integration and assessment of a model for shape memory alloys considering multiaxial nonproportional loading cases. Int. J. Solids Struct. 54, 28-99.

Hallai, J.F., Kyriakides, S., 2013. Underlying material response for lüders-like instabilities. Int. J. Plast. 47, 1-12.

Halphen, B., Nguyen, Q.S., 1975. Sur les matériaux standard généralisés. J. Mecanique 14, 39-63.

Iadicola, M.A., Shaw, J.A., 2004. Rate and thermal sensitivities of unstable transformation behavior in a shape memory alloy. Int. J. Plast. 20, 577-605.

Jiang, D., Kyriakides, S., Bechle, N.J., Landis, C.M., 2017a. Bending of pseudoelastic NiTi tubes. Int. J. Solids Struct. 124, 192-214. 
Jiang, D., Kyriakides, S., Landis, C.M., 2017b. Propagation of phase transformation fronts in pseudoelastic niti tubes under uniaxial tension. Extrem Mech. Letters 15, 113-121.

Jiang, D., Kyriakides, S., Landis, C.M., Kazinakis, K., 2017c. Modeling of propagation of phase transformation fronts in NiTi under uniaxial tension. Eur. J. Mech. A 64, 131-142.

Jirásek, M., Rolshoven, S., 2003. Comparison of integral-type nonlocal plasticity models for strain softening materials. Int. J. Eng. Sci. 41, 1553-1602.

Lagoudas, D.C., Hartl, D.J., Chemisky, Y., Machado, L.G., Popov, P., 2012. Constitutive model for the numerical analysis of phase transformation in polycrystalline shape memory alloys. Int. J. Plast. 32-33, 155-183.

Mao, S.C., Luo, J.F., Zhang, Z., Wub, M.H., Liu, Y., Han, X.D., 2010. EBSD studies of the stressinduced B2-B19' martensitic transformation in NiTi tubes under uniaxial tension and compression. Acta Mater. 58, 3357-3366.

Matsumoto, O., Miyazaki, Sand Otsuka, K., Tamura, H., 1987. Crystallography of martensitic transformation in Ti-Ni single crystals. Acta Mettal. 35, 2137-2144.

Mehrabi, R., Kadkhodaei, M., Elahinia, M., 2014. Constitutive modeling of tension-torsion coupling and tension-compression asymmetry in NiTi shape memory alloys. Smart Mater. Struct. 23, 075021.

Mielke, A., Theil, F., 2004. On rate-independent hysteresis models. NODEA-Nonlinear. Diff. 11, 151-189. doi:10.1007/s00030-003-1052-7.

Mohd Jani, J., Leary, M., Subic, A., Gibson, M.A., 2014. A review of shape memory alloy research, applications and opportunities. Mater. Des. 56, 1078-1113.

Mori, T., Tanaka, K., 1973. Average stress in the matrix and average elastic energy of materials with misfitting inclusions. Acta Mettal. 21, 571-574.

Otsuka, K., Wayman, C.M., 1998. Shape Memory Materials. Cambridge University Press.

Peerlings, R., Geers, M., De Borst, R., Brekelmans, W., 2001. A critical comparison of nonlocal and gradient-enhanced softening continua. Int. J. Solids Struct. 38, 7723-7746.

Peng, X., Pi, W., Fan, J., 2008. A microstructure-based constitutive model for the pseudoelastic behavior of NiTi SMAs. Int. J. Plast. 24, 966-990.

Pieczyska, E., Tobushi, H., Kulasinski, K., 2013. Development of transformation bands in TiNi SMA for various stress and strain rates studied by a fast and sensitive infrared camera. Smart Mater. Struct. 22, 035007.

Pouya, M., Elibol, C., Wagner, M.F.X., 2017. Understanding complex stress states in pseudoelastic shape memory alloys: macroscopic modeling considering localization and tension-compression asymmetry, in: ASM International - International Conference on Shape Memory and Superelastic Technologies, SMST 2017, pp. 167-168.

Razaee-Hajidehi, M., Tuma, K., Stupkiewicz, S., 2019. Gradient-enhanced thermomechanical 3D model for simulation of transformation patterns in pseudoelastic shape memory alloys. Int. J. Plast., in press. 
Reedlunn, B., Churchill, C.B., Nelson, E.E., Shaw, J.A., Daly, S.H., 2014. Tension, compression, and bending of superelastic shape memory alloy tubes. J. Mech. Phys. Solids 63, 506-537.

Reedlunn, B., Daly, S., Shaw, J.A., 2012. Tension-torsion experiments on superelastic shape memory alloy tubes, in: ASME 2012 Conference on Smart Materials, Adaptive Structures and Intelligent Systems, SMASIS 2012, pp. 213-222.

Reedlunn, B., Shaw, J.A., Daly, S.H., 2017. Axial-torsion behavior of superelastic NiTi tubes, in: SMST 2017: Conference Proceedings from the International Conference on Shape Memory and Superelastic Technologies May 15-19, 2017, San Diego, California, USA, ASME.

Sadjadpour, A., Bhattacharya, K., 2007. A micromechanics-inspired constitutive model for shapememory alloys. Smart Mater. Struct. 16, 1751-1765.

Sedlák, P., Frost, M., 2018. Numerical simulations of NiTi shape memory alloy wire behaviors in tension, compression, and torsion. Acta Phys. Pol. A 134, 842-846.

Sedlák, P., Frost, M., Benešová, B., Šittner, P., Ben Zineb, T., 2012. Thermomechanical model for NiTi-based shape memory alloys including R-phase and material anisotropy under multi-axial loadings. Int. J. Plast. 39, 132-151.

Sedmák, P., Pilch, J., Heller, L., Kopeček, J., Wright, J., Sedlák, P., Frost, M., Šittner, P., 2016. Grain-resolved analysis of localized deformation in nickel-titanium wire under tensile load. Science $353,559-562$.

Shaw, J.A., Kyriakides, S., 1995. Thermomechanical aspects of NiTi. J. Mech. Phys. Solids 43, $1243-1281$.

Shaw, J.A., Kyriakides, S., 1997a. Initiation and propagation of localized deformation in elasto-plastic strips under uniaxial tension. Int. J. Plast. 13, 837-871.

Shaw, J.A., Kyriakides, S., 1997b. On the nucleation and propagation of phase transformation fronts in a niti alloy. Acta Mater. 45, 683-700.

Šittner, P., Liu, Y., Novák, V., 2005. On the origin of lüders-like deformation of NiTi shape memory alloys. J. Mech. Phys. Solids 53, 1719-46.

Šittner, P., Novák, V., 2000. Anisotropy of martensitic transformations in modeling of shape memory alloy polycrystals. Int. J. Plast. 16, 1243-1268.

Stebner, A.P., Brinson, 2013. Explicit finite element implementation of an improved three dimensional constitutive model for shape memory alloys. Comput. Methods Appl. Mech. Eng. 257, 17-35.

Stupkiewicz, S., Petryk, H., 2013. A robust model of pseudoelasticity in shape memory alloys. Int. J. Numer. Methods Eng. 93, 747-769.

Sun, Q.P., Li, Z.Q., 2002. Phase transformation in superelastic NiTi polycrystalline micro-tubes under tension and torsion - from localization to homogeneous deformation. Int. J. Solids Struct. 39, 3797-3809.

Watkins, R., Reedlunn, B., Daly, S.H., Shaw, J.A., 2018. Uniaxial, pure bending, and column buckling experiments on superelastic NiTi rods and tubes. Int. J. Solids Struct. 146, 1-28. 
Xiao, Y., Zeng, P., Lei, L., 2017. Grain size effect on mechanical performance of nanostructured superelastic NiTi alloy. Mater. Res. Express 4, 035702.

Young, M.L., Wagner, M.F.X., Frenzel, J., Schmahl, W.W., Eggeler, G., 2010. Phase volume fractions and strain measurements in an ultrafine-grained NiTi shape-memory alloy during tensile loading. Acta Mater. 58, 2344-2354.

Zhang, S., He, Y., 2018. Fatigue resistance of branching phase-transformation fronts in pseudoelastic NiTi polycrystalline strips. Int. J. Solids Struct. 135, 233-244.

Zhang, X., Feng, P., He, Y., Yu, T., Sun, Q., 2010. Experimental study on rate dependence of macroscopic domain and stress hysteresis in niti shape memory alloy strips. Int. J. Mech. Sci. 52, 1660-1670. 
This figure "3Dribbon-VFM-evol.png" is available in "png" format from: http://arxiv.org/ps/1912.01356v1 
This figure "3DtubeBEND-ALL-evol.png" is available in "png" format from: http://arxiv.org/ps/1912.01356v1 